\title{
Long Covid: A Review on Cardiovascular Disease (CVD) in Post-Acute Sequalae of SARS-CoV-2 Infection (PASC)
}

Scopus Author ID 56708804600

Received: 19.03.2021; Revised: 10.05.2021; Accepted: 15.05.2021; Published: 13.08.2021

\begin{abstract}
Coronavirus disease 2019 (COVID-19) caused by the virus SARS-CoV-2 was declared a pandemic by the World Health Organization (WHO) in March 2020. The clinical spectrum of COVID19 can range from asymptomatic to severe symptoms and even death. Recent studies show that a growing number of patients with COVID-19 will experience prolonged symptoms (Post-Acute Sequalae of SARS-CoV-2), which now days known as "Long Covid". A team of researchers from Italy reported that nearly 9 in 10 patients discharged were still experiencing at least one symptom 60 days after infection. Symptoms reported are fatigue, dyspnea, joint pain, chest pain, and other uncommon symptoms. Through ACE2 surface receptor, SARS-CoV-2 has been linked to cardiovascular events. As PASC symptoms vary widely, cardiovascular sequelae need to be considered through lifetime management. Therefore, this review aims to summarize an update of the long covid effect on Cardiovascular Disease (CVD), the impacts, and Post-Acute Sequalae of SARS-CoV-2 Infection (PASC) management.
\end{abstract}

Keywords: COVID-19; Post-Acute Sequelae of SARS-CoV-2 Infection; Long Covid; cardiovascular disease.

(C) 2021 by the authors. This article is an open-access article distributed under the terms and conditions of the Creative Commons Attribution (CC BY) license (https://creativecommons.org/licenses/by/4.0/).

\section{Introduction}

The infection through the Coronavirus originated in the Wuhan city of China's Hubei Province by the end of 2019 [1]. In March 2020, the Coronavirus disease 2019 (COVID-19), caused by the virus SARS-CoV-2, was declared a pandemic by the World Health Organization (WHO) [2]. Since then, there have been confirmed COVID-19 reports in 219 Countries and Territories around the world. Almost similar to its predecessor, SARS, the symptoms of COVID-19 make infected people feel with symptoms like the flu ones [3]. During this time, studies have shown that the clinical spectrum of COVID-19 can range from asymptomatic infection to mild upper respiratory tract illness to severe interstitial pneumonia with respiratory failure and even death. It is estimated that non-severe patients with no symptoms or mild symptoms could represent around 30-60\% of all infections [4,5]. However, COVID-19 is a new disease, and uncertainties remain regarding the possible long-term sequelae of the infection after recovery. Patients with severe COVID-19 symptoms, especially those requiring mechanical ventilators during hospitalization, have developed long-term complications and have incomplete recovery after discharge [6]. Although SARS-CoV-2 primarily affects the lungs, it has been observed that several other organs, including the kidney, can also be affected. 


\section{Profile and Timeline Definitions}

As many COVID-19 signs and symptoms can occur during and after the infection, the National Institute for Health and Care Excellence (NICE) has provided a guideline on the clinical definition for the profiles and timelines [7]. These are the three clinical definitions; Acute COVID-19: COVID-19 signs and symptoms for up to 4 weeks. Ongoing Symptomatic COVID-19: COVID-19 signs and symptoms from 4 to 12 weeks. Post-COVID-19 is COVID19 signs and symptoms for more than 12 weeks and are not explained by an alternative diagnosis.

Recent studies show that a growing number of patients with COVID-19 will experience prolonged symptoms, in which the exact profiles and timelines remain uncertain [8,9]. Early in the course of the pandemic, patients identified this trend, referring to themselves as "LongHaulers" and the prolonged illness as "Long COVID" [10]. Therefore, this review aims to summarize an update of the long covid effect on Cardio Vascular Disease (CVD), the impacts, and Post-Acute Sequelae of SARS-CoV-2 Infection (PASC) management.

\section{Long COVID-19}

Weeks and months after the onset of Acute COVID-19, people may continue to suffer. “Long COVID” was first used by Elisa Perego from Lombardy in May 2020. It is being used to describe illness in people who have recovered from COVID-19 but still report lasting effects of the infection or have had the usual symptoms for far longer than expected.[10,11]. It is still unknown why some people's recovery is prolonged [12].

A team of researchers from Italy reported that nearly 9 in 10 patients $(87.4 \%)$ discharged from a Rome hospital after recovering from COVID-19 were still experiencing at least one symptom 60 days after onset. They found that $13 \%$ of the 143 people were completely free of any symptoms, while $32 \%$ had one or two symptoms, and 55\% had three or more [13]. Symptoms reported are fatigue (53\%), dyspnoea (43\%), joint pain (27\%), and chest pain (22\%). Two-fifths of patients reported a worsened quality of life [11,13]. A prior study showed 78 out of 100 patients who had recovered from COVID-19 had abnormal cardiovascular MRI findings, and 36 of them reported unusual fatigue and dyspnoea [5].

A study cohort from Jin Yin-tan Hospital (Wuhan, China) reported 76\% of patients after 6 months of illness onset have at least one symptom that persisted. More than $50 \%$ of patients presented with residual chest imaging abnormalities. At 6 months after symptom onset, fatigue or muscle weakness and sleep difficulties were the main symptoms of patients who had recovered from COVID-19 [14]. Studies from Renmin Hospital of Wuhan University show that $49.6 \%$ of patients who had recovered from COVID-19 still had one or more common symptoms, including physical decline, fatigue, and myalgia, 3 months after discharge [15].

From the clinical perspective, the COVID-19 incubation period and clinical phase involve 3 weeks. The post-acute-COVID can be described as the illness extending beyond three weeks from the onset of first symptoms, and the chronic COVID-19 as extending beyond 12 weeks. The diagnosis of "Long COVID" or "Long-Haulers" should be considered if various symptoms and signs linger well beyond the period of convalescence in COVID-19 [7].

\section{Impact of Covid-19 on Cardiovascular Disease}

The genetic factors and pathways related to COVID-19 involve the angiotensinconverting enzyme 2 (ACE2) gene encoding the ACE2 surface receptor associated with the 
SARS-CoV-2 virus [16]. ACE2 is localized in various human organs, including oral and nasal mucosa, nasopharynx, lung, and heart [17]. In the renin-angiotensin-aldosterone system (RAAS), ACE2 catalyst angiotensin II's conversion to angiotensin 1-7, which acts as a vasodilator, exerts a protective effect in the cardiovascular system [18,41]. Elevated angiotensin II in COVID-19 patients has been linked to vascular hyperpermeability and severe acute lung injury, as well as hypocalcemia and a possible sign of hyperaldosteronism [19-21].

Moreover, this mechanism is possibly explaining the increased prevalence of cardiac and renal complications. An additional pathophysiological explanation for the extent of cardiovascular involvement stems from the systemic inflammatory effects driven by the virus, predominantly through cytokine release and inflammatory cascade highlighted by serum biomarker elevation [22]. The expression of the ACE2 receptor on myocytes, coronary endothelial cells, and arterial smooth muscle increases the risk of organ damage in individuals with COVID-19, as the virus uses these receptors to gain entry into cells [23-25]. The prior study has shown that among hospitalized patients, cardiac injury is a common condition to find with a higher risk of in-hospital mortality [26]. This may be related to the ACE2 acting receptor for SARS-CoV-2 [27,28]. The possible mechanisms explaining this association include cytokine storm, microangiopathy, viral myocarditis, stress-induced cardiomyopathy, classic myocardial infarction due to infection-induced atherosclerotic plaque instability. All these mechanisms have a common denominator as endothelial injury [29,30].

As the most common pre-existing condition, CVD might have potentially exacerbated the severity of COVID-19. COVID-19 is mostly a lung disease. Initial local damage can be followed by an intense and relatively late cytokine storm originating from the imbalance of $\mathrm{T}$ cells' activation with dysregulated release of interleukin (IL)-6, IL-17, and other cytokines [31]. In parallel, COVID-19 can also begin with signs of severe coronary artery disease or myocarditis in the absence of a history of cardiovascular diseases or the presence of isolated cardiovascular risk factors $[19,20,32]$.

The cardiac injury mechanism is not well established. Still, it likely involves increased cardiac stress due to respiratory failure and hypoxemia, direct myocardial infection by SARS$\mathrm{CoV}-2$, indirect injury from the systematic inflammatory response, or a combination of all 3 factors. Due to direct infection or global inflammation, dysfunction in cardiac pericytes and endothelial cells can disrupt coronary microcirculation with downstream ischemic consequences. Still, the relationship to COVID-19 is purely conjectural [33]. SARS-CoV-2 effects on microvascular function, notably pericyte injury, particularly in heart failure patients, have also been reported [23]. Besides, a drug used to treat COVID-19 patients has cardiovascular side effects [34].

The major studies have focused on acute complications, but long-term sequelae may be possible based on the above mechanism. One hypothesized that following cardiovascular sequelae and complications may develop in individuals with COVID-19 and pre-exciting cardiovascular risk factors: acute coronary syndrome, myocardial infarction, arrhythmia, cardiogenic shock, heart failure, myocarditis, and venous thromboembolism [22]. The study found that $13 \%$ of patients had a cardiovascular sequel, the most important of which was increased resting heart rate. Besides, seven patients reported a recent diagnosis of hypertension after COVID-19's long-term damage to the cardiovascular system [15]. 


\section{The Social Impact of PASC}

As a global pandemic with unprecedented medical, economic, and social consequences, COVID-19 has been potentially severe cardiovascular complications and sequelae [26]. Patients with pre-existing CVD also suffer worse outcomes with COVID-19 infection. Beyond these direct consequences, COVID-19 has reshaped the delivery of cardiovascular care - noncritical and elective procedures are postponed, and distancing imperatives have led to rapid scaling of telemedicine and cancellation of cardiac rehabilitation. The repeated public health messages that those with chronic conditions should practice social and physical distancing may adversely affect the health-seeking behavior of patients with CVD [35,36].

Through the "Long Covid" cardiovascular symptoms, morbidity and mortality due to CVD might increase. Furthermore, long-term studies are required to determine the incidence and clinical course of myocardial damage caused by COVID-19 to implement a routine cardiac imaging screening that allows for the treatment of post-COVID-19 heart syndrome [37].

\section{PASC Management}

According to the Office for National Statistics, one out of five patients with positive for Covid-19 testing exhibit symptoms for five weeks and more This provides challenges for determining best-practice on the standard of care [38]. To assist clinicians, the National Institute for Health and Care Excellence (NICE), the Scottish Intercollegiate Guidelines Network (SIGND), and the Royal College of General Practitioners (RCGP) have developed the "COVID-19 rapid guideline: managing the long-term effects of COVID-19". The guideline covers care for people who experience Covid-19 signs and symptoms for more than four weeks which is not explained by other alternative diagnoses[38]. The guidelines provide information to help the public understand their symptoms and to recognize when to seek help. With the guidelines, physicians can educate people who were suspected or confirmed with acute Covid19 on what they might expect during their recovery time.

A person with symptoms 3 or more weeks after the Covid-19 onset needs to get a clinical assessment through full history, current symptom, and clinical testing that may be considered. Blood tests can pinpoint the causes of continuing symptoms and exclude conditions like pulmonary embolism or myocarditis [39]. Patient comorbidities, including diabetes, hypertension, kidney disease, or ischemic heart disease, need to be managed in conjunction with covid-19 treatment [39]. After all, medical management will be parallel with selfmanagement. Support from social, financial, and cultural may be needed to help "LongHaulers" survived all the symptoms of PASC [3,40].

\section{Conclusions}

"Long Covid" is a new medical term to describe the experience of prolonged symptoms of Covid-19 infections. As PASC symptoms vary widely, cardiovascular sequelae need to be considered through lifetime management. Since chest pain is common in PASC, further clinical priority needs to separate musculoskeletal and other non-specific chest pain from serious cardiovascular conditions. Even though there are not yet definitive evidence-based recommendations for the management of PASC, the physician can use the NICE guideline to approach patient symptoms through better quality of life. 


\section{Funding}

The authors declare that they didn't receive any fund.

\section{Acknowledgments}

The authors like to thank their affiliated universities for partially supporting this work: Trisakti University and Al-Nahrain University.

\section{Conflicts of Interest}

The authors declare no conflict of interest.

\section{References}

1. Monajjemi, M.; Shahriari, S.; Mollamin F. Evaluation of Coronavirus Families \& Covid-19 Proteins: Molecular Modeling Study. Biointerface Res. Appl. Chem. 2020, 10, 6039-57, https://doi.org/10.33263/BRIAC105.60396057.

2. Imran, A.; Alharbi, O.M.L. COVID-19: Disease, management, treatment, and social impact. Sci Total Environ. 2020, https://doi.org/10.1016/j.scitotenv.2020.138861.

3. de Sire, A.; Andrenelli, E.; Negrini, F.; Negrini, S.; Ceravolo, M.G. Systematic rapid living review on rehabilitation needs due to COVID-19: update as of April 30th, 2020. Eur J Phys Rehabil Med. 2020, 56, 35460, https://doi.org/10.23736/S1973-9087.20.06329-7.

4. Mizumoto, K.; Kagaya, K.; Zarebski, A.; Chowell, G.; Estimating the asymptomatic proportion of coronavirus disease 2019 (COVID-19) cases on board the Diamond Princess cruise ship, Yokohama, Japan. Euro Surveill. 2020, 25, 1-5, https://doi.org/10.2807/1560-7917.ES.2020.25.10.2000180.

5. Puntmann, V.O.; Carerj, M.L.; Wieters, I. Outcomes of cardiovascular magnetic resonance imaging in patients recently recovered from coronavirus disease 2019 (COVID-19). JAMA Cardiol 2020, 5, 1265-73, https://doi.org/10.1001/jamacardio.2020.3557.

6. Cortivonis, M.; Perico, N.; Remuzzi, G. Long-term follow-up of recovered patients with COVID-19. The Lancet 2021, 397, 173-75, https://doi.org/10.1016/S0140-6736(21)00039-8.

7. Nikhra, V. Living with 'long COVID-19': The long-term complications and sequelae. Int J Clin Virol. 2021, 5, 011-017, https://doi.org/10.29328/journal.ijcv.1001030.

8. Arnold, D.T.; Hamilton, F.W.; Milne, A.; Morley, A.; Viner, J.; Attwod, M. Patient outcomes after hospitalisation with COVID-19 and implications for follow-up; results from a prospective UK cohort. medRxiv. 2021, 76, 399-401, https://doi.org/10.1136/thoraxjnl-2020-216086.

9. Michelen, M.; Manoharan, L.; Elkheir, N.; Cheng, V.; Dagens, D.; Hastie, C. Characterising long-term covid19: a rapid living systematic review. medRxiv. 2020, https://doi.org/10.1101/2020.12.08.20246025.

10. Callarad, F.; Perego E. How and why patients made Long Covid. Soc Sci Med. 2021, 268, https://doi.org/10.1016/j.socscimed.2020.113426.

11. Mahase, E. Covid-19: What do we know about "long covid"?. The BMJ. 2020, 370, https://doi.org/10.1136/bmj.m2815.

12. Wu, F.; Wang, A.; Liu, M. Neutralizing antibody responses to SARS-CoV-2 in a COVID-19 recovered patient cohort and their implications. medRxiy. 2020, https://doi.org/10.1101/2020.03.30.20047365.

13. Carfì, A.; Bernabei, R.; Landi. F.G. Against COVID-19 Post-Acute Care Study Group. Persistent symptoms in patients after acute covid-19. JAMA. 2020, 324, 603-5, https://doi.org/10.1001/jama.2020.12603.

14. Huang, C.; Huang, L.; Wang, Y.; Li, X.; Ren, L.; Gu, X. 6-month consequences of COVID-19 in patients discharged from hospital: a cohort study. Lancet 2021, 397, 220-232, https://doi.org/10.1016/S01406736(20)32656-8.

15. Quitang, X.; Ming, X.; Jiao L.; Yinghui, L.; Jixiang, Z.; Yu, X.; Clinical sequelae of COVID-19 survivors in Wuhan, China: a single-centre longitudinal study. Clinical Microbiology and Infection. 2021, 27, 89-95, https://doi.org/10.1016/j.cmi.2020.09.023.

16. Wu, B.B.; Gu, D.Z.; Yu, J.N.; Yang, J.; Shen, W.Q. Association between ABO blood groups and COVID-19 infection, severity and demise: A systematic review and meta-analysis. Infect Genet Evol. 2020, 84, 104485, https://doi.org/10.1016/j.meegid.2020.104485. 
17. Hamming, I.; Timens, W.; Bulthuis, M.L.; Lely, A.T.; Navis, G.; van Goor, H. Tissue distribution of ACE2 protein, the functional receptor for SARS coronavirus. A first step in understanding SARS pathogenesis. $J$. Pathol. 2004, 203, 631-637, https://doi.org/10.1002/path.1570.

18. Kuster, G.M.; Pfister, O.; Burkard, T.; Zhou, Q.; Twerenbold, R.; Haaf, P.; Widmer, A.F.; Osswald, S. SARSCoV2: Should inhibitors of the renin-angiotensin system be withdrawn in patients with COVID-19? Eur. Heart J. 2020, https://doi.org/10.1093/eurheartj/ehaa235.

19. Clerkin, K.J.; Fried, J.A.; Raikhelkar, J.; Sayer, G.; Griffin, J.M.; Masoumi, A.; Jain, S.S.; Burkhoff, D.; Kumaraiah, D.; Rabbani, L.; Schwartz, A.; Uriel, N. COVID-19 and cardiovascular disease. Circ. 2020, 141, 1648-1655, https://doi.org/10.1161/CIRCULATIONAHA.120.046941.

20. Liu, Y.; Yang, Y.; Zhang, C.; Huang, F.; Wang, F.; Yuan, J.; Wang, Z.; Li, J.; Li, J.; Feng, C.; Zhang, Z.; Wang, L.; Peng, L.; Chen, L.; Qin, Y.; Zhao, D.; Tan, S.; Yin, L.; Xu, J.; Zhou, C.; Jiang, C.; Liu, L. Clinical and biochemical indexes from 2019-nCoV infected patients linked to viral loads and lung injury. Sci China Life Sci. 2020, 63, 364-74, https://doi.org/10.1007/s11427-020-1643-8.

21. Mai, F.; Pinto R.D.; Ferri, C. COVID-19 and cardiovascular disease. Jour of Cardiol. 2020, 76, 453-8, https://doi.org/10.1016/j.jjcc.2020.07.013.

22. Driggin, E.; Madhaven, M.V.; Bikdeli, B.; Chuich, T.; Laracy, J.; Bondi-Zoccai, G. Cardiovascular considerations for patients, health care workers, and health systems during the Coronavirus Disease 2019 (COVID-19) pandemic. J Am Coll Cardiol. 2020, 75, 2352-71, https://doi.org/10.1016/j.jacc.2020.03.031.

23. Chen, L.; Li, X.; Chen, M.; Feng, Y.; Xiong, C. The ACE2 expression in human heart indicates new potential mechanism of heart injury among patients infected with SARS-CoV-2. Cardiovasc Res. 2020, 116, 1097 1100, https://doi.org/10.1093/cvr/cvaa078.

24. Cormican, D.S.; Winter, D.; McHugh, S.; Sonny, A.; Crowley, J.; Yu, R.; Barrack, F.; Nunez-Gil, I.; Ramakrishna, H. Severe acute respiratory syndrome coronavirus-2 cardiovascular complications: implications for cardiothoracic anesthesiology. J Cardiothorac Vasc Anesth. 2020, 35, 932-43, https://doi.org/10.1053/j.jvca.2020.05.035.

25. Guo, J.; Huang, Z.; Lin, L.; Lv, J. Coronavirus disease 2019 (COVID-19) and cardiovascular disease: a viewpoint on the potential influence of angiotensin-converting enzyme inhibitors/angiotensin receptor blockers on onset and severity of severe acute respiratory syndrome coronavirus 2 infection. J Am Heart Assoc. 2020, 9, https://doi.org/10.1161/JAHA.120.016219.

26. Fried, J.A.; Ramasubbu, K.; Bhatt, R.; Topkara, V.K.; Clerkin, K.J.; Horn, E.; Rabbani, L.; Brodie, D.; Jain, S.S.; Kirtane, A.;J.; Masoumi, A.; Takeda, K.; Kumaraiah, D.; Burkhoff, D.; Leon, M.; Schwartz, A.; Uriel, N.; Sayer, G. The variety of cardiovascular presentations of COVID-19. Circ. 2020, 141, 1930-6, https://doi.org/10.1161/CIRCULATIONAHA.120.047164.

27. Demertzis, Z.D.; Dagher, C.; Malette, K.M.; Fadel, R.A.; Bradley, P.B.; Brar, I. Cardiac sequelae of novel coronavirus disease 2019 (COVID-19): a clinical case series. Eur Heart J Case Rep. 2020, 4, 1-6, https://doi.org/10.1093/ehjcr/ytaa179.

28. South, A.M.; Diz, D.I.; Chappell, M.C. COVID-19, ACE2, and the cardiovascular consequences. Am J Physiol Heart Circ Physiol. 2020, 318, 1084-90, https://doi.org/10.1152/ajpheart.00217.2020.

29. Tersalvi, G.; Vicenzi, M.; Calabretta, D.; Biasco, L.; Pedrazzini, G.; Winterton, D. Elevated troponin in patients with coronavirus disease 2019: possible mecha- nisms. J Card Fail. 2020, 26, 470-475, https://doi.org/10.1016/j.cardfail.2020.04.009.

30. Varga, Z.; Flammer, A.; Steiger, P. Endothelial cell infection and endotheliitis in COVID-19. The Lancet 2020, 395, 1417-1418, https://doi.org/10.1016/S0140-6736(20)30937-5.

31. Siddiqi, H.K.; Mehra, M.R. COVID-19 illness in native and immunosuppressed states: a clinical-therapeutic staging proposal. J Heart Lung Transplant. 2020, 39, 405-407, https://doi.org/10.1016/j.healun.2020.03.012

32. Zhang, P.; Zhu, L.; Cai, J.; Lei, F.; Qin, J.; Xie, J. Association of inpatient use of angiotensin converting enzyme inhibitors and angiotensin II receptor blockers with mortality among patients with hypertentions hospitalized with COVID-19. Circ Res. 2020, 126, 1671-81, https://doi.org/10.1161/CIRCRESAHA.120.317134.

33. Akhmerov, A.; Marban, E. Covid-19 and the Heart. Circ Res AHA. 2020, 126, 1443-55, https://doi.org/10.1161/CIRCRESAHA.120.317055.

34. Gori,T.; Lelieveld, J.; Munzel, T. Perspective: cardiovascular disease and the Covid-19 pandemic. Basic Res Cardiol. 2020, 115, 32-5, https://doi.org/10.1007/s00395-020-0792-4. 
35. Garcia, S.; Albaghdadi, M.S.; Meraj, P.M. Reduction in ST-segment elevation cardiac catheterization laboratory activations in the United States during COVID-19 pandemic. J Am Coll Cardiol. 2020, 75, 28712, https://doi.org/10.1016/j.jacc.2020.04.011.

36. Lim, S.L.; Woo, K.L.; Lim, E.; Ng, Faclin.; Chan, M.Y.; Gandhi, M. Impact of COVID-19 on helath-related quality of life in patients with cardiovascular disease: a multi-ethnic Asian study. Health Qual Life Outcomes. 2020, 18, 387-93, https://doi.org/10.1186/s12955-020-01640-5.

37. Gasecka A, Pruc M, Kukula K, Gilis-Malinowska N, Filipiak KJ, Jaguszewski MJ, Szarpak L. Post-COVID19 heart syndrome. Cardiol J. 2021;28(2):353-354. doi: 10.5603/CJ.a2021.0028.

38. Shah, W.; Hillman, T.; Playford, E.D.; Hishmeh, L. Managing the long term effects of covid-19: summary of NICE, SIGN, and RCGP rapid guideline. BMJ. 2021, 372, 136, https://doi.org/10.1136/bmj.n136.

39. Greenhalgj, T.; Knight, M.; A’Court, C.; Buxton, M.; Husain, L. Management of post-acute covid-19 in primary care. The BMJ. 2020, 370, 1-7, https://doi.org/10.1136/bmj.m3026.

40. Mukaino, M.; Tatemoto, T.; Kumazawa, N. Staying active in isolation: telerehabilitation for individuals with the severe acute respiratory syndrome coronavirus 2 infection. Am J Phys Med Rehabil. 2020, 99, 478-9, https://doi.org/10.1097/PHM.0000000000001441.

41. Hadi, A.G.; Kadhom, M.; Hairunisa, N.; Yousif, E. Mohammed Salam A. A Review on COVID-19: Origin, Spread, Symptoms, Treatment, and Prevention. Biointerface Res. Appl Chem. 2020, 10, 7234-42, https://doi.org/10.33263/BRIAC106.72347242. 